\title{
Subject Assigned to Arm But Not Treated
}

National Cancer Institute

\section{Source}

National Cancer Institute. Subject Assigned to Arm But Not Treated. NCI Thesaurus. Code C142238.

The subject, who met eligibility criteria, was assigned to an arm but was not treated. 